\title{
Incertitude, rationalité et confiance dans les choix d'investissement : Une analyse de la relation entre marchés financiers et capital-risque
}

Eric Nasica et Dominique Dufour

\section{(2) OpenEdition}

Journals

Édition électronique

URL : https://journals.openedition.org/rei/2223

DOI : $10.4000 /$ rei.2223

ISSN : 1773-0198

Éditeur

De Boeck Supérieur

Édition imprimée

Date de publication : 15 septembre 2007

Pagination : 103-124

ISSN : 0154-3229

Référence électronique

Eric Nasica et Dominique Dufour, «Incertitude, rationalité et confiance dans les choix

d'investissement: Une analyse de la relation entre marchés financiers et capital-risque », Revue

d'économie industrielle [En ligne], 119 | 3e trimestre 2007, mis en ligne le 15 septembre 2009, consulté le 02 juin 2022. URL : http://journals.openedition.org/rei/2223 ; DOl : https://doi.org/10.4000/rei.2223

Ce document a été généré automatiquement le 2 juin 2022

(c) Revue d'économie industrielle 


\title{
Incertitude, rationalité et confiance dans les choix d'investissement: Une analyse de la relation entre marchés financiers et capital-risque
}

\author{
Eric Nasica et Dominique Dufour
}

\section{NOTE DE L'AUTEUR}

Les auteurs remercient les rapporteurs anonymes de leurs remarques et suggestions.

\section{Introduction}

1 La littérature consacrée aux déterminants du capital-risque insiste sur la relation positive existant entre le montant des fonds investis dans cette activité et la situation prévalant sur les marchés financiers. Ceci n'est pas surprenant dans la mesure où des anticipations d'introductions en bourse favorables, synonymes de plus-values importantes pour les capital-risqueurs, sont des incitations essentielles pour le marché du capital-risque. Cependant, au cours des dernières années, la relation entre marchés financiers et activité de capital-risque apparaît beaucoup moins claire: les comportements récents d'investissement des capital-risqueurs semblent s'être sensiblement et durablement déconnectés de l'évolution des marchés financiers. Nous avons choisi d'expliquer ce phénomène en fondant notre analyse sur un élément essentiel mais généralement négligé dans la littérature: la forme spécifique de rationalité adoptée par les capital-risqueurs dans leurs choix d'investissement.

Dans la première partie de l'article (section 1), nous présentons les arguments théoriques et empiriques qui fondent l'existence d'une relation entre la situation prévalant sur les marchés financiers et les investissements en capital-risque. 
3 Dans un second temps (section 2), nous rappelons les particularités de l'environnement décisionnel dans lequel évoluent les intervenants sur le marché du capital-risque. C'est cet environnement décisionnel spécifique qui conditionne le choix des outils analytiques appropriés à l'étude de la rationalité des sociétés de capital-risque.

4 Nous présentons ensuite (section 3) un modèle, inspiré des approches en termes de rationalité limitée, formalisant les comportements d'investissement des sociétés de capital-risque. Ce modèle met en évidence les raisons pour lesquelles une société de capital-risque peut, en adoptant certaines règles de décisions rationnelles en situation d'incertitude, modifier sensiblement le volume et la forme de ses investissements au cours du temps. Plus précisément, ce modèle caractérise les comportements des capital-risqueurs par deux traits distinctifs : le premier est une sur-réaction de la part des intervenants sur le marché du capital-risque qui les conduit à diminuer brutalement et sensiblement la pondération du facteur marchés financiers dans leur processus de décision d'investissement; le deuxième est une rigidification des comportements des capital-risqueurs conduisant ces derniers à répéter de période en période le même type de comportement en matière d'investissement, induisant par là même une déconnexion entre les choix d'investissement et l'évolution des performances du marché financier.

5 Enfin, nous analysons de manière statistique les caractéristiques de la relation entre l'évolution des marchés financiers et l'investissement en capital-risque sur la période récente (1999-2006) en nous basant sur le cas de la France. Les résultats statistiques obtenus sont ensuite interprétés à la lumière du modèle théorique que nous proposons (section 4).

\section{I. - La relation entre marchés financiers et capital- risque}

6 Le capital-risque est l'une des composantes du capital-investissement. Il est possible, au sens large, de considérer toute activité d'apport de fonds propres à des sociétés non cotées comme des opérations de capital-investissement. Le capital-investissement regroupe trois grandes rubriques: le capital-risque, le capital-développement et le capital-reprise/transmission d'entreprise. Le capital-développement et le capitalreprise/transmission d'entreprise concernent le financement de firmes ayant déjà plusieurs années d'existence : elles ont dépassé la phase de démarrage et se situent en phase de croissance, voire de maturité ${ }^{1}$.

7 Le capital-risque au sens strict, sur lequel est centré cet article, est réservé aux opérations d'investissement dans des entreprises en création ou dans de jeunes entreprises technologiques. Ce type de financement fait intervenir trois métiers bien distincts. Le premier est le seed capital ou capital d'amorçage : c'est le financement très en amont du démarrage de l'entreprise. L'objectif de cette levée de fonds est de prouver la faisabilité de l'idée ou du projet. La finalité de cette étape est de permettre l'élaboration du plan de développement ou plan d'affaires (business plan) pour convaincre des investisseurs de financer le lancement d'un nouveau projet. Le second métier du capital-risque est le capital-création. C'est le financement du démarrage proprement dit (start up) de l'entreprise. Il concerne le «premier tour de table » et les premières années de vie de l'entreprise. La troisième et dernière activité formant le 
capital-risque est le financement de la phase de post-création ou phase de première croissance (early stage). Elle concerne les deux ou trois premières années de démarrage de l'entreprise. Elle va jusqu'au stade du lancement industriel et commercial du projet.

Pour mieux comprendre l'influence des marchés financiers sur l'activité de capitalrisque, il est primordial d'expliciter ce que Gompers et Lerner (2001) appellent le " cycle du capital-investissement ». Ce cycle comprend une succession de trois grandes phases. Il débute par une levée de fonds du capital-risqueur auprès d'agents économiques désireux d'investir dans des entreprises non cotées. Une deuxième phase correspond à l'investissement dans l'entreprise proprement dit. L'apport de fonds propres s'accompagne de la mise en place par le capital-risqueur de procédures de contrôle ${ }^{2}$ visant à limiter le risque de son portefeuille ainsi que les conflits d'intérêt susceptibles de naître dans le cadre de la relation d'agence l'unissant aux dirigeants de l'entreprise financée. Les opérations de sortie constituent la dernière phase du cycle du capital-investissement. Le capital-risqueur dispose de différents modes de sortie: la cession de ses parts aux dirigeants ou aux cadres de l'entreprise, à un industriel ou à un autre financier, la liquidation de l'entreprise (en cas d'échec), et enfin l'introduction en bourse. Les éventuelles plus-values réalisées par le capital-risqueur lors de cette phase permettent, d'une part, de rémunérer les fonds investis dans l'organisme de capitalrisque et, d'autre part, de réinvestir dans de futures participations. Un nouveau cycle de capital-investissement peut alors redémarrer.

9 La phase de sortie est donc cruciale puisqu'elle permet de mesurer le montant de valeur créée durant la période de présence du capital-risqueur dans le capital de l'entreprise et d'évaluer la pertinence des processus de sélection et de contrôle qu'il a mis en place.

Notre analyse est centrée sur l'une des possibilités de sortie mentionnée ci-dessus : les marchés financiers. Les raisons de ce choix sont multiples. Tout d'abord, de nombreuses études économétriques mettent en évidence une influence importante des marchés financiers sur l'investissement en capital-risque. Ces études utilisent différents facteurs pour illustrer le rôle des marchés financiers. Ainsi, pour Black et Gilson (1988), Gompers et Lerner (1998) et Jeng et Wells (2000), les IPO (initial public offerings), qui reflètent le rendement potentiel du capital-risque, apparaissent comme l'un des déterminants essentiels des investissements. Black et Gilson en concluent que le développement du marché du capital-investissement est indissociable de l'existence de marchés financiers développés et profonds capables d'absorber les introductions en bourse des entreprises. D'autres études montrent que la liquidité du marché, représentée notamment par la capitalisation boursière (Schertler, 2003) a un impact positif sur le développement des investissements en capital-risque. Enfin, plus récemment, Gompers et alii (2005) ont souligné, dans une étude portant sur les ÉtatsUnis, que l'activité de capital-risque était très sensible aux signaux, tels que le $Q$ de Tobin, émis par les marchés financiers.

11 Une autre raison importante nous incitant à privilégier les introductions en bourse est que ce mode de sortie est le plus rémunérateur pour le capital-risqueur. Une étude de Venture Economics (1988) montre ainsi que la sortie sous forme d'IPO est cinq fois plus rentable que la sortie via la cession des parts à une autre entreprise ${ }^{3}$. Les travaux plus récents de Gompers (1995) pour les États-Unis et de Schwienbacher (2004) pour l'Europe, confirment les meilleures performances de la sortie en bourse par rapport à la sortie industrielle. Par conséquent, le fait pour un capital-risqueur de mener à bien des IPO lui permet de signaler son expérience au marché et, partant, de réduire les coûts de 
transaction de sa relation avec les dirigeants. Dans le même ordre d'idées, le fait de réaliser des introductions en bourse réussies améliore la réputation du capital-risqueur et lui permet de lever des fonds auprès des investisseurs à des conditions plus favorables.

Enfin, la sortie sous forme d'IPO permet aux capital-risqueurs de mieux gérer les éventuels risques de conflit d'intérêt avec les dirigeants (Black et Gilson, 1988). En effet, lors de l'introduction en bourse, les dirigeants-fondateurs gardent souvent une influence importante dans l'entreprise. À l'inverse, lorsque le capital-risqueur sort du capital via une sortie industrielle ou en cédant ses parts à un autre fonds d'investissement, l'indépendance des dirigeants n'est plus assurée. La perspective de restitution du contrôle va réduire les comportements opportunistes et inciter les dirigeants à mener à bien le projet de développement, la création de valeur et, in fine, l'introduction en bourse.

13 Les arguments théoriques et empiriques rappelés ci-dessus mettent donc clairement en évidence le fait que les investissements en capital-risque dépendent fortement de la situation prévalant sur les marchés financiers.

\section{II. - Capital-risque, incertitude radicale et rationalité limitée}

Les firmes recourant au capital-risque possèdent des caractéristiques spécifiques qui rendent leur activité particulièrement incertaine.

En premier lieu, elles sont fréquemment positionnées sur des marchés en fort développement, émergents, voire à créer au moment où l'entreprise est financée. En second lieu, leurs produits se situent généralement soit dans la phase de conception soit dans la phase de lancement ${ }^{4}$. Troisièmement, l'activité des start-up technologiques est fondée sur la mise en œuvre et la gestion d'actifs immatériels (savoir-faire, développement technologique) qui jouent un rôle primordial dans l'essor de la firme mais sont difficilement quantifiables et négociables. Or, ces actifs immatériels, à la différence d'actifs physiques, peuvent difficilement servir de " collatéraux » au sens de garanties tangibles qui jouent en général le rôle de réducteurs d'incertitude et d'asymétrie d'information dans les relations de financement ${ }^{5}$.

L'environnement décisionnel des capital-risqueurs n'est donc pas simplement risqué mais incertain ${ }^{6}$. Ce constat a des implications importantes sur le choix des outils analytiques appropriés à l'étude de la relation capital-

risqueur/start-up technologique. L'une des voies explorées au cours des dernières années consiste à analyser cette relation par le biais des outils de la théorie des options réelles. L'intérêt d'une telle démarche est indéniable.

En premier lieu, les options réelles sont considérées comme un outil d'analyse permettant de pallier certaines insuffisances des méthodes traditionnelles d'évaluation des projets d'investissement (Dixit et Pindyck, 1994, Trigeorgis, 1996). Ces dernières (comme l'approche DCF, discounted cash-flows), basées sur l'actualisation, impliquent un biais lorsqu'elles sont appliquées dans l'environnement décisionnel particulièrement incertain entourant les start-up technologiques. En effet, plus le risque est élevé, plus le taux d'actualisation augmente et plus la valeur actuelle de l'entreprise sera faible. Dans ce contexte, les projets (et les entreprises) à très forts risques sont pénalisés alors que 
l'on sent intuitivement qu'il n'est pas tenu compte de la valeur stratégique des opportunités de croissance associées à ces projets. Une autre faiblesse des méthodes d'évaluation traditionnelles est que seules les possibilités présentes du décideur sont prises en compte. Cela conduit à adopter une vision "statique» de la décision d'investissement qui ignore d'éventuelles interventions des dirigeants à des dates ultérieures en réaction à l'évolution de l'environnement et à l'acquisition d'informations nouvelles.

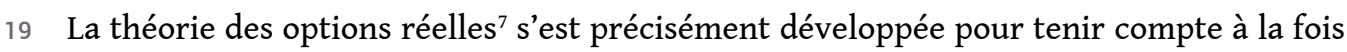
de l'irréversibilitée, de la flexibilité et du caractère dynamique des projets d'investissement.

20 En effet, un projet d'investissement contient un certain nombre d'options. Il s'agit notamment d'»options d'abandon" lorsque l'entreprise a la possibilité de ne pas poursuivre indéfiniment un projet qui s'avérerait non rentable. Il s'agit également d'»options de croissance ». Celles-ci reposent sur l'idée qu'en investissant dans une technologie nouvelle, l'entreprise acquiert principalement l'option d'investir dans une série de projets futurs. Ainsi, pour une start-up technologique, les investissements réalisés pendant la phase de démarrage (par exemple en R\&D) engendrent des opportunités de croissance qui représentent l'essentiel de la valeur de l'entreprise. Enfin, un projet contient l'option de pouvoir attendre ou échelonner les investissements et, le cas échéant, reporter la décision. On l'appelle l'» option de flexibilité ». Sa valeur est d'autant plus élevée que la nature irréversible du projet est marquée.

21 Une autre caractéristique intéressante de l'approche par les options réelles est qu'elle permet de rendre compte de certaines pratiques utilisées par les capital-risqueurs. En effet, afin de réduire les risques inhérents aux financements des projets innovants, les organismes de capital-risque procèdent à un financement "par étapes" (stage financing) consistant à fractionner l'investissement et à intervenir en fonction de l'évolution de l'environnement et de la réalisation des différents objectifs intermédiaires (milestones) fixés à la fois par le capital-risqueur et le dirigeant. Comme le note Dubocage (2003), ces investissements successifs ont le statut d'»options d'apprentissage » : ils donnent des informations à l'investisseur sur la rentabilité du projet et réduisent l'incertitude? .

22 Malgré les atouts indéniables de l'approche par les options réelles, cette méthode est très peu utilisée par les capital-risqueurs (Desbrières et Broye, 2000) en raison de son degré de complexité technique (lié notamment à la difficulté à déterminer la valeur de certains paramètres comme le prix du sous-jacent). D'un point de vue théorique, cette méthode se heurte également à un problème de contradiction interne (Dubocage, 2003; Dubocage et Rivaud-Danset, 2006). En effet, en se fixant comme objectif de proposer une règle de décision fondée sur un résultat chiffré, elle a recours à des méthodes (actualisation des cash-flows futurs ${ }^{10}$, référence à des marchés financiers efficients, etc.) finalement très proches de celles de l'analyse financière traditionnelle. Or, ces méthodes sont difficilement applicables dans le cadre de l'environnement d'incertitude radicale caractérisant la relation entre capital-risqueurs et start-up technologiques.

Cette remarque nous conduit à analyser les comportements des investisseurs en capital-risque avec les outils analytiques fournis par les approches en termes de rationalité «limitée» ou «procédurale» plutôt que par les approches plus 
traditionnelles fondées sur la rationalité «substantielle » (selon la terminologie de Simon, 1976).

Dans l'hypothèse de rationalité substantielle, largement utilisée dans la théorie néoclassique de la décision, l'ensemble des états de la nature, c'est-à-dire l'ensemble des actions et de leurs conséquences possibles, est prédéterminé et connu par l'individu au moment de la prise de décision. Celui-ci est également supposé doté de capacités cognitives et calculatoires illimitées, ce qui lui permet d'associer des probabilités objectives ou subjectives (Savage, 1954) à l'occurrence de chacun de ces événements possibles, de calculer l'utilité espérée qu'il retirerait de chacune des conséquences possibles de ses actions et de choisir la décision qui lui apporterait le plus de satisfaction possible.

L'approche en termes de rationalité procédurale s'applique en revanche à un environnement décisionnel radicalement différent. L'hypothèse selon laquelle les individus connaissent parfaitement la liste des événements futurs potentiels est considérée comme irréaliste et ce, pour deux raisons.

La première est la faiblesse des capacités cognitives de l'individu qui limite son accès à l'information et l'empêche de disposer d'une connaissance exhaustive de l'ensemble des événements possibles (Stigler, 1961, Williamson, 1994). La seconde est que la liste des états de la nature possibles n'est pas considérée comme une donnée préalable à la prise de décision. À l'instar de Shackle (1955, p. 6), pour qui les agents économiques prennent des décisions "cruciales» et ne peuvent donc "chasser de leur esprit la possibilité que le simple fait d'exécuter une expérience peut définitivement détruire les circonstances dans lesquelles elle a été accomplie», le raisonnement en termes de rationalité procédurale tient compte du fait que les décisions successives des individus produisent des irréversibilités qui modifient l'éventail des événements possibles (Simon, 1976). Il est alors difficile d'associer des probabilités, fussent-elles subjectives, à des événements futurs qui peuvent être inconnus car non encore existants au moment de la prise de décision : la maximisation d'une utilité espérée et la détermination d'une solution « optimale » deviennent alors impossibles.

Dans cet environnement d'incertitude radicale et de capacités cognitives limitées dans lequel les capital-risqueurs doivent prendre leurs décisions, c'est un autre mode de prise de décision que le traditionnel comportement d'optimisation qui prévaut. Il est fondé sur la mise en place et l'utilisation de procédures conscientes - on parle de règles de comportement (rules of thumb) - ou inconscientes - on les qualifie d'habitudes (Hodgson, 1988) qui permettent aux agents économiques de prendre des décisions rapidement sans avoir à tenir compte de la totalité de l'information disponible.

Loin d'être des défaillances du marché, ces procédures sont rationnelles parce qu'« elles sont des modes de comportement que la firme (ou l'individu) élabore afin de prendre des décisions dans un environnement complexe d'incertitude et d'information imparfaite " (Cyert et Simon, 1983, p. 105). Les exemples concrets de leur utilisation foisonnent dans le domaine économique et financier: période d'amortissement pour la décision d'investissement, taux normal d'utilisation de la capacité, ratios "normaux» d'endettement, de liquidité et de fonds propres, etc.

29 Les modèles de rationalité limitée ont suivi différentes voies afin de formaliser les décisions des agents économiques en information imparfaite ${ }^{11}$. Parmi ces travaux, le 
modèle de fiabilité développé par Heiner (1983) nous semble particulièrement adapté à l'analyse des décisions d'investissement des sociétés de capital-risque.

L'argumentation de Heiner est la suivante: il existe un écart entre les capacités cognitives d'un agent et la complexité de l'environnement auquel il est confronté [competence-difficulty ( (C-D ») gap]. Cet écart découle des changements imprévus qui affectent l'environnement ainsi que des capacités limitées des agents à réagir de manière appropriée à ces changements. C'est cet écart qui introduit l'incertitude dans l'analyse des choix.

Or, selon Heiner, c'est précisément cette incertitude, sous la forme du C-D gap, qui est à l'origine des régularités et donc de la prévisibilité des comportements économiques. Un agent économique, dans un environnement décisionnel de simple risque probabilisable et agissant conformément à l'hypothèse d'anticipations rationnelles (Muth, 1961) aurait un comportement extrêmement irrégulier car, étant totalement flexible dans ses choix, il réagirait immédiatement à toute perturbation de l'environnement afin de se replacer dans l'état optimal. En revanche, chez Heiner, la rigidité - et donc la prévisibilité - des comportements viennent de la nécessité de comparer le risque d'erreur lié au choix d'une nouvelle action avec le coût d'opportunité de la conservation de l'ancienne ligne de conduite.

En d'autres termes, plus le degré d'incertitude est élevé, plus le risque de prendre la mauvaise décision (c'est-à-dire de décider de changer les procédures de choix en vigueur et encourir des pertes subséquentes, ou décider de ne pas changer ces procédures alors qu'une modification aurait engendré des gains) est élevé. Si les agents avaient une connaissance parfaite de leur environnement, ils seraient toujours capables de déterminer si le moment est propice pour modifier leur comportement. Cependant, en situation d'incertitude et de connaissance incomplète, les agents économiques reçoivent des signaux qu'ils interprètent difficilement. Il n'est, par conséquent, intéressant de modifier son comportement habituel que si cette modification engendre des gains espérés substantiels ; c'est-à-dire lorsque les gains associés au changement de comportement (ou les gains nets comparés à la situation consistant à ne pas modifier son comportement) et leur probabilité sont élevés.

Ce raisonnement a une implication importante : un agent doit ignorer les actions qui ne s'avèrent appropriées que dans des situations rares ou " anormales ». Inversement, le répertoire d'un agent doit se limiter aux actions qui sont adaptées à des situations relativement probables ou récurrentes. Ainsi, ce répertoire exclut-il des actions qui sont optimales dans certaines situations, et ce même si la probabilité de réalisation de ces situations est positive.

Le modèle que nous développons ci-dessous afin d'expliquer les comportements d'investissement des capital-riqueurs reprend, en les prolongeant, les grandes lignes de l'approche de Heiner ${ }^{12}$.

\section{III. - Le modèle}

\subsection{Notations et conditions de fiabilité}

Considérons une société de capital-risque (SCR dans la suite) initialement limitée dans son répertoire d'actions possibles en matière d'investissement dans les start-up technologiques. Supposons, pour simplifier, qu'il y a initialement une seule action 
possible dans le répertoire : «Investir dans les phases de développement et de reprisetransmission des start-up technologiques ». Phase de démarrage. Cette condition traduit le fait que le capital-risqueur introduit une nouvelle action (« investir dans la phase de démarrage de start-up technologiques ») dans son répertoire d'actions possibles quand le gain espéré, si l'environnement est favorable au choix de cette action, est supérieur à la perte envisagée lorsque l'environnement est défavorable, avec:

$52 \rho=(r / w)$ (probabilité de faire le bon choix/probabilité de faire le mauvais choix) représente le ratio de fiabilité associé à la décision d'investissement c'est-à-dire la confiance qu'accorde la SCR à son choix d'investissement dans une phase de démarrage d'une start-up technologique. 
53 fiabilité doit atteindre afin que la SCR décide d'élargir son répertoire d'action à l'investissement dans la phase de démarrage d'une start-up technologique.

cette perspective, si $\rho>T$ la SCR aura un comportement "flexible » ou " accommodant» vis-à-vis des demandes de financement des start-up en phase de démarrage. En revanche, si $\rho<T$ elle aura un comportement "rigide» consistant à n'investir que dans des firmes technologiques matures sous forme de capitaldéveloppement et de capital-reprise/transmission.

\subsection{Dynamique du modèle}

Nous allons maintenant examiner les facteurs qui influencent chacun des côtés de l'inégalité représentant la condition de fiabilité afin de comprendre les raisons pour lesquelles une SCR peut évoluer d'un comportement "rigide " à un comportement «flexible » face aux demandes de financement des start-up technologiques.

Le côté droit de l'inégalité dépend de la probabilité $(1-\Pi)$, c'est-à-dire de la probabilité que les circonstances soient défavorables pour investir dans la phase de démarrage de start-up technologiques.

Pour mieux comprendre la nature particulière de cette probabilité, il faut d'abord soigneusement la distinguer de la probabilité de perte sur un portefeuille d'investissement telle qu'elle est traditionnellement mesurée par les capital-risqueurs. Lorsqu'on raisonne sur un portefeuille de capital-risque donné, il est possible d'estimer avec un niveau de confiance et de précision acceptables le risque de perte ainsi que les corrélations entre les investissements de ce portefeuille afin de constituer des provisions adéquates pour absorber les pertes espérées. Il y a d'ailleurs une forte incitation à effectuer convenablement ce type d'évaluation : une sous-estimation du risque entraînant de fortes pertes potentielles pour le capital-risqueur, tandis qu'une surestimation du risque (et un sous-investissement) se traduira par des pertes de parts de marché tout aussi néfastes.

En revanche, l'état de la connaissance relatif à la détermination de la probabilité (1 - П) est beaucoup moins complet. Les capital-risqueurs savent seulement qu'il existe une faible probabilité que se produise un choc défavorable aux conséquences potentiellement catastrophiques pour le marché du capital-risque dans son ensemble. Néanmoins, ils ne disposent ni d'une connaissance probabiliste a priori de $(1-\Pi)$ ni de suffisamment de données pour estimer cette probabilité sur la base de séries statistiques passées. Ce niveau d'ignorance s'apparente davantage à de l'incertitude au sens de Keynes (1937) ou de Knight (1921) qu'à un simple risque probabilisable.

On peut alors difficilement supposer que, en ce qui concerne l'estimation de $(1-\Pi)$, les probabilités subjectives des investisseurs en capital-risque convergeront vers les probabilités objectives. Ce constat est encore renforcé par le fait que, puisqu'un choc défavorable de grande ampleur (un type krach boursier ou tout autre phénomène de grande ampleur susceptible de remettre en cause la stabilité du système dans son ensemble) se produit rarement, il peut être négligé, sans dommage pour les décideurs, pendant de très longues périodes. Dans ces conditions, le fait pour un capital-risqueur de sous-estimer le niveau effectif de $(1-\Pi)$ dans l'évaluation du risque de son portefeuille au cours de périodes «normales » (i.e. hors périodes de crises) d'activité 
économique ne relève pas d'un comportement irrationnel. Une société de capital-risque qui essayerait d'intégrer dans le calcul de son taux de rentabilité exigé une prime de risque appropriée à l'existence d'événements peu fréquents mais générateurs de fortes pertes potentielles adopterait un comportement relativement « rigide» vis-à-vis du financement des firmes technologiques en création. Elle se priverait ainsi, contrairement à des investisseurs plus "flexibles", de parts de marché et de gains potentiels importants durant la phase de «boom » de l'investissement. De plus, en cas de crise importante sur le secteur concerné (en l'occurrence, les start-up technologiques en phase de démarrage), un grand nombre de sociétés de capital-risque " flexibles » sont touchées, de sorte que la responsabilité de l'échec n'incombe à aucune d'entre elles en particulier. Cela contraste fortement avec la situation prévalant durant le boom d'investissement où les capital-risqueurs « exagérément rigides » enregistrent de manière isolée des résultats inférieurs aux autres investisseurs. On se trouve ainsi dans une situation de "structure de récompense asymétrique » où les comportements «flexibles » sont récompensés davantage et sanctionnés dans une moindre mesure que les comportements $«$ rigides $»^{13}$.

La nature de la probabilité $(1-\Pi)$ se prête donc difficilement à un traitement analytique fondé sur les outils traditionnels de la théorie de la décision ${ }^{14}$. En revanche, certains travaux relevant de la psychologie cognitive ont étudié en profondeur les méthodes simplifiées (heuristiques) mises en place par les individus pour estimer les probabilités, telles qu' $(1-\Pi)$, d'événements peu fréquents et singuliers n'obéissant pas à une loi de probabilité connue.

61 L'une de ces heuristiques est l'"heuristique de disponibilité " (availability heuristics) (Kahneman et Tversky, 1982). Elle est employée lorsque les agents estiment la probabilité d'occurrence de certains événements à partir de la mémorisation qu'ils ont d'événements similaires. Dans ce contexte, la probabilité subjective de réalisation d'un événement est d'autant plus forte que sa fréquence passée est élevée ou que sa dernière date de réalisation est proche de la période courante. L'exemple qui illustre le mieux ce phénomène est celui du comportement d'un conducteur qui vient d'assister à un accident de la route. La réponse immédiate du conducteur est de conduire beaucoup plus prudemment. Il se comporte ainsi comme si la probabilité d'avoir un accident avait soudainement augmenté. Mais, progressivement, à mesure que le temps s'écoule, l'image de l'accident s'estompe dans la mémoire et la prudence du conducteur diminue.

Dans le cadre de notre modèle, l'accident de la route va prendre la forme d'une défaillance brutale et aiguë d'un indicateur de performance du marché financier. Une telle défaillance s'assimile à un choc défavorable pris en compte dans la formation des probabilités subjectives par les capital-risqueurs. Le raisonnement en termes d'heuristique de disponibilité, que nous supposons utilisé par les sociétés de capitalrisque, implique alors que ces dernières "sur-réagissent » de telle manière que la probabilité subjective de réalisation d'un environnement défavorable $(1-\Pi)$ atteigne un niveau élevé, éventuellement supérieur à la probabilité $(1-\Pi)_{\mathrm{E}}$ effective (mais inconnue a priori par les agents).

Quelles sont les conséquences de cette modification des anticipations des SCR sur la condition de fiabilité du modèle?

Tout d'abord, la hausse de la probabilité $(1-\Pi)$ engendre une hausse de la limite de tolérance T. Si cette hausse est suffisamment importante, la condition de fiabilité s'écrit : $\rho<T$ 

éventuelles corrélations existant entre l'évolution des marchés financiers et le montant des investissements sur le marché du capital-risque français. Nous interprétons ensuite les résultats statistiques obtenus à la lumière du modèle théorique que nous venons de présenter.

\subsection{L'analyse statistique}

70 L'analyse statistique du capital-risque français soulève plusieurs difficultés. La principale concerne le montant des fonds levés ainsi que des sorties. Il est impossible de disposer de statistiques globales à vocation d'exhaustivité, les formes d'intervention en capital-risque sont trop variées pour le permettre. Il faut donc se contenter des statistiques compilées par des organismes privés ou publics par sondage auprès des organismes professionnels du capital-risque. Les statistiques que nous présentons ont donc plus pour objet de donner une représentation des tendances à l'œuvre que de décrire de manière complète la réalité du capital-risque en France depuis 1999.

71 Afin d'analyser statistiquement la relation entre l'évolution des marchés financiers et l'investissement en capital-risque, nous utilisons des données relatives à l'indice ITCAC et aux levées de fonds dans le secteur internet. Ce secteur a, entre 1999 et 2001, reçu une part importante des levées de fonds de la période ${ }^{15}$. Ce constat a été fait sur la même période outre-Atlantique (Lerner 2002). de sociétés technologiques. En décembre 2006, il comprenait 76 valeurs dont plusieurs étaient cotées sur le nouveau marché quand celui-ci existait encore. Cet indice est 
disponible depuis le 1er janvier 1999. Nous avons retenu l'ITCAC dans la mesure où il s'agit d'un indice étroitement lié au financement des valeurs technologiques. Par conséquent, un renversement de tendance de ce type de marché voué au financement des entreprises technologiques ne peut que freiner le financement par capital-risque. Nous avons préféré l'ITCAC à l'indice du Nouveau Marché dans la mesure où figuraient sur ce dernier marché des entreprises que l'on ne pouvait pas rattacher aux entreprises technologiques. Ajoutons que l'ITCAC est encore aujourd'hui disponible ce qui n'est pas le cas de l'Indice du Nouveau Marché.

La période étudiée est comprise entre le 1er janvier 1999 et le 30 septembre 2006, soit 93 observations mensuelles.

Afin de pouvoir mettre les deux statistiques à la même échelle, nous avons représenté l'indice ITCAC divisé par 1000 et les levées de fonds internet mesurées en millions d'euros. Les levées de fonds qui sont disponibles sur le Journaldunet, ont été désaisonnalisées par utilisation des moyennes mobiles. Au démarrage de la période, les deux statistiques sont voisines.

Pour ce qui concerne l'ITCAC, le graphique appelle les commentaires suivants :

- les levées de fonds ont connu sur la période une instabilité plus élevée que l'ITCAC,

- sur la période 1999-2002, les évolutions des deux statistiques semblent obéir à la même dynamique avec une amplitude plus marquée pour les levées de fonds,

- à partir de janvier 2003, nous assistons à une tendance à la progression de l'ITCAC alors même que les levées de fonds sont caractérisées par une forte irrégularité.

\section{Graphique - Évolution de m'ITCAC et des levées de fonds internet}
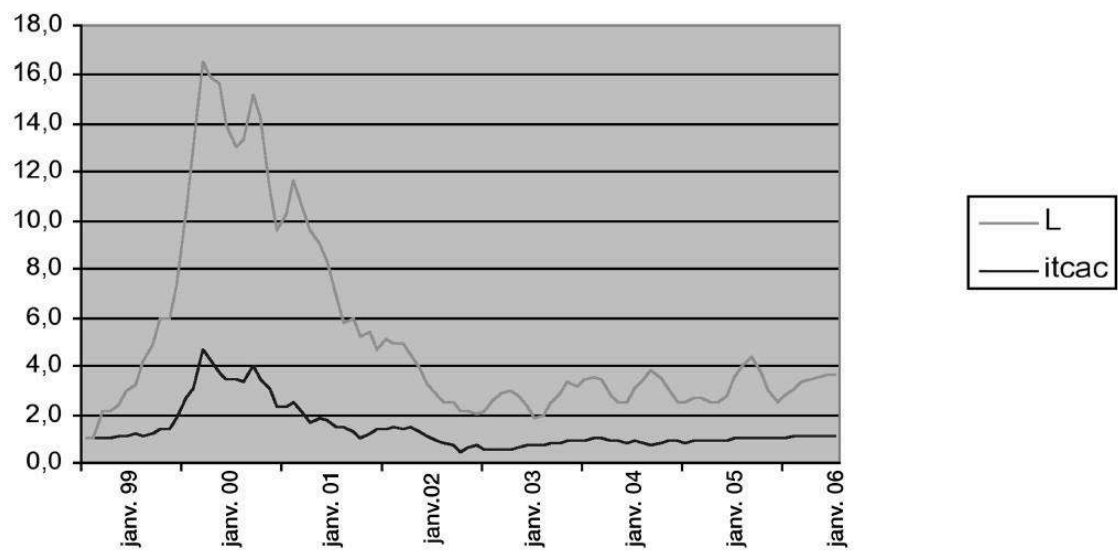

Source : Journaldunet.org

La relation entre l'évolution des marchés financiers et l'investissement en capitalrisque est testée par une régression linéaire de la forme :

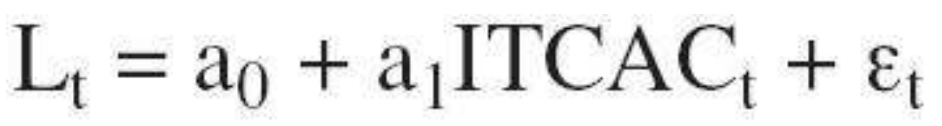

La variable expliquée, notée Lt, est le montant d'investissement en capital-risque au sein des entreprises du secteur internet le mois t. ITCACt est la valeur de l'ITCAC pour ce même mois. 
Nous avons tout d'abord testé la régression sur la période 1999-2006.

Tableau 1 - Régression sur la période 1999-2006

\begin{tabular}{|l|l|l|l|}
\hline & Constante & ITCAC & DW \\
\hline $\mathrm{a}$ & $-0,63$ & 3,15 & 0,586 \\
\hline $\mathrm{t}$ & $-3,55$ & 29,4 & \\
\hline Probabilité & 0,000 & 0,000 & \\
\hline
\end{tabular}

Les résultats de la régression (tableau 1) font apparaitre une autocorrélation des résidus statistiquement significative. Les résultats sont donnés dans le tableau 2.

Tableau 2 - Régression AR sur la période 1999-2006

\begin{tabular}{|l|l|l|l|l|}
\hline & Constante & ITCAC & AR(1) & DW \\
\hline $\mathrm{a}$ & 1,57 & 1,65 & 0,93 & 1,646 \\
\hline $\mathrm{t}$ & 1,60 & 7,416 & 25,46 & $\mathrm{R}^{2}$ \\
\hline Probabilité & 0,113 & 0,000 & 0,000 & 0,924 \\
\hline
\end{tabular}

Il apparaît donc bien sur l'ensemble de la période une relation statistiquement significative entre les levées de fonds et l'indice ITCAC. Nous avons testé différents rangs d'auto-corrélation, seule l'auto-corrélation de rang 1 est statistiquement significative.

81 Nous avons ensuite testé la stabilité de cette relation en décomposant la période globale en deux sous-périodes : 1999-2002 et 2003-2006. Nous donnons les résultats de ces corrélations dans les tableaux 3 et 4 ci-dessous.

Tableau 3 - Régression AR sur la période 1999-2002

\begin{tabular}{|l|l|l|l|l|}
\hline & Constante & ITCAC & AR(1) & DW \\
\hline $\mathrm{a}$ & 2,55 & 1,72 & 0,91 & 1,89 \\
\hline $\mathrm{t}$ & 2,08 & 6,24 & 16,27 & $\mathrm{R}^{2}$ \\
\hline Probabilité & 0,048 & 0,000 & 0,000 & 0,945 \\
\hline
\end{tabular}

82 Les résultats obtenus pour la période 1999-2002 (tableau 3) sont voisins de ceux observés sur la période entière. Là encore, seule l'auto-corrélation de rang 1 est statistiquement significative. 
83 L'examen des résultats de la régression sur la période 2003-2006 (tableau 4) fait apparaître des résultats profondément différents.

Tableau 4 - Régression AR sur la période 2003-2006

\begin{tabular}{|l|l|l|l|l|l|}
\hline & Constante & ITCAC & AR(1) & AR(2) & DW \\
\hline $\mathrm{a}$ & 1,91 & 0,18 & 1,29 & $-0,79$ & 2,03 \\
\hline $\mathrm{t}$ & 4,85 & 0,43 & 11,92 & $-7,42$ & $\mathrm{R}_{-}$ \\
\hline Probabilité & 0,000 & 0,670 & 0,000 & 0,000 & 0,793 \\
\hline
\end{tabular}

Si la relation entre les levées de fonds et l'ITCAC est significative avant 2003, elle cesse de l'être à partir de janvier 2003. En d'autres termes, les levées de fonds restent insensibles à la progression de l'indice à compter de cette date. Les levées de fonds sont fortement auto-corrélées avec des auto-corrélations de rangs 1 et 2 statistiquement significatives et de sens contraire.

\subsection{L'interprétation des résultats à la lumière du modèle}

En rapprochant les résultats de l'analyse statistique à ceux de notre modèle théorique, il est possible de mettre en évidence une rupture dans les comportements d'investissement des capital-risqueurs au cours des dernières années.

Tout d'abord, au cours de la période 1999-2002, l'ITCAC est une variable explicative fortement significative traduisant une influence très sensible du marché financier des valeurs technologiques sur l'investissement en capital-risque. Les raisons à l'adoption de ce comportement sont néanmoins différentes selon la sous-période considérée. En effet, entre 1999 et 2000, c'est-à-dire avant le e-krach, la corrélation positive entre marché financier et investissement s'explique par une hausse de la confiance $\rho$ due à l'amélioration de l'indice ITCAC. De son côté, le seuil minimum du ratio de fiabilité, T, est faible car les capital-risqueurs, en l'absence de chocs défavorables récents, estiment que la probabilité de réalisation d'un événement défavorable sur le marché du capitalrisque est peu élevée. La condition de fiabilité de notre modèle est donc telle que $\rho>T$. La situation est alors particulièrement favorable à l'essor de l'activité de financement des start-up technologiques en phase de démarrage en raison du comportement " accommodant» des capital-risqueurs à leur égard. En revanche, au cours de la deuxième sous-période, entre 2000 et 2002, immédiatement après le e-krach, les effets de l'adoption d'une heuristique de disponibilité par les capital-risqueurs se font pleinement ressentir: ils entraînent une augmentation brutale et aiguë du seuil minimum $\mathrm{T}$ en raison de la «sur-réaction » à la hausse affectant la probabilité (1-ח). Comme, de son côté, la confiance $\rho$ des capital-risqueurs diminue fortement en raison de l'effondrement de l'ITCAC, il s'ensuit logiquement un retournement de la condition de fiabilité du modèle qui devient telle que $\rho \ll$ T. Cela se traduit concrètement par une réallocation des portefeuilles des sociétés de capital-risque au détriment des investissements dans les start-up en phase de démarrage qui subissent ainsi un fort rationnement. 
87 À partir de 2003, l'ITCAC n'est plus une variable explicative significative: malgré l'évolution à nouveau favorable de cet indice observée depuis janvier 2003, l'investissement en capital-risque au sens strict ne redémarre pas et l'essentiel des fonds continue à être orienté vers les entreprises plus matures, essentiellement sous forme de capital-reprise/transmission. En d'autres termes, le comportement d'investissement des sociétés de capital-risque semble s'être déconnecté de l'évolution du marché financier des valeurs technologiques. Notre modèle fournit une explication à cette rupture dans les comportements : malgré les évolutions récentes favorables de l'ITCAC qui ont contribué à améliorer la confiance $r$ depuis le début de l'année 2003, le souvenir du e-krach reste encore présent dans les esprits des investisseurs. D'après le modèle, l'effet-mémoire associé à l'heuristique de disponibilité utilisée par les capitalrisqueurs maintient leur estimation de la probabilité (1-ח) - et donc le seuil minimum du ratio de fiabilité, $\mathrm{T}$ - à un niveau élevé. Dans ces conditions, en dépit de la hausse récente de $\rho$, la condition de fiabilité ne s'est pas retournée et demeure telle que $\rho<T$. On se trouve ainsi dans une situation où l'offre des capital-risqueurs est peu flexible vis-à-vis des demandes de financement des start-up en phase de démarrage et où l'évolution du marché financier ne joue plus un rôle déterminant dans les décisions d'investissement.

Les chiffres récents confirment le caractère durable de cette rigidité dans les comportements des investisseurs qui se traduit par le maintien d'une très faible proportion de leurs portefeuilles investie dans l'activité de capital-risque au sens strict. Ainsi, en France, les investissements en capital-risque (phase d'amorçage et de création) ne représentent en 2005 que $6 \%$ (481 millions d'euros) de l'ensemble du capital-investissement contre $20 \%$ lors du point haut historique de 2000 . Les opérations de LBO/capital-transmission (6,3 milliards d'euros) représentant quant à elle la plus grande partie (75\%) des investissements (AFIC, 2006).

\section{Conclusion}

L'étude des comportements d'investissement des capital-risqueurs au cours de la période récente est riche d'enseignements dans le cadre de l'élaboration d'une analyse des décisions de financement en information imparfaite. Les caractéristiques spécifiques aux start-up technologiques soulignent la nécessité de développer une approche théorique prenant explicitement en compte la forte incertitude pesant sur l'activité des firmes technologiques en phase de démarrage et donc indirectement sur les sociétés de capital-risque. Les décisions d'investissement de ces dernières se prêtent alors difficilement à une analyse recourant aux outils traditionnels de l'optimisation mais n'en sont pas pour autant irrationnelles. La forme différente de rationalité qui est à l'œuvre est intéressante à plus d'un titre.

90 Elle permet d'intégrer dans l'analyse des choix d'investissement en capital-risque des éléments souvent sous-estimés ou ignorés dans la plupart des approches empiriques ou théoriques ${ }^{16}$. Parmi ces éléments omis dans la littérature, nous avons mis l'accent sur la confiance et les procédures heuristiques particulières utilisées par les investisseurs en situation d'incertitude. Toutes ces notions sont, comme nous l'avons montré, centrales car elles expliquent à la fois l'existence de rigidités (et de stabilité) dans les comportements mais aussi le caractère brutal et intense des changements d'opinion des 
capital-risqueurs (et plus généralement des investisseurs en situation d'incertitude) sur la viabilité de leurs choix d'investissement.

Elles permettent également de mieux comprendre - c'était l'objectif essentiel de ce travail - la déconnexion observée ces dernières années entre le montant des investissements et l'un des indicateurs économiques habituellement considérés comme essentiels dans l'activité de capital-risque, l'évolution des marchés financiers.

\section{BIBLIOGRAPHIE}

Adam M.C. et Farber A. (1994), « Le financement de l'innovation technologique : théorie économique et expérience européenne », PUF, Paris.

AFIC (2005, 2006), « Rapports sur l'activité du capital-investissement en France », Études AFIC et Pricewaterhousecoopers.

Amram M. et Kulatilaka N. (1999), « Real Options : Managing Strategic Investment in an Uncertain World », Harvard Business School Press, Boston.

Black B.-S. et Gilson R.-J., « Venture Capital and the Structure of Capital Markets : Banks versus Stock Markets »; Journal of Financial Economics, March 1998, vol. 47, iss. 3, pp. 243-77.

Berger N. et Udell F (1998), « The economics of small business finance : The roles of private equity and debt markets in the financial growth cycle » Journal of Banking and Finance, vol. 22(6-8), pp. 613-673.

Booz, Allen et Hamilton (1968), « Management of New Products », New York, Booz, Allen \& Hamilton Inc.

Cyert R.-M., et Simon H.-A. (1983), "The behavioral approach : With emphasis on economics ", Behavioral Science, 28(2), 95-108.

Daudé B. (1981), « Analyse de la Maîtrise des Risques », Fondation Nationale pour l'Enseignement de la Gestion des Entreprises, Chotard, Paris.

Desbrières P. et Broye G., " Critères d'évaluation des investisseurs en capital : le cas français ", Finance, Contrôle, Stratégie, vol. 3, n 3, septembre 2000, pp. 5-43.

Dixit A. et Pindyck R. (1994), «Investment Under Uncertainty », Princeton Universtity Press, Princeton.

Dubocage, E. (2003), « Les options réelles : un outil théorique adapté à l'évolution des start-up financées par le capital-risque ? », Document de travail du CEPN, n² 2003-12.

Dubocage E. et Rivaud-Danset D. (2006), « Le capital-risque », coll. Repères, La Découverte, Paris.

Giudici G. et Roosenboom P. (2004), «Venture Capital And New Stock Markets In Europe » in « The Rise and Fall of Europe's New Stock Markets », G. Giudici et P. Roosenboom (eds), in "Advances in Financial Economics », vol. 10, pp. 1-24, Oxford; Amsterdam and San Diego : Elsevier. 
Gompers P., Kovner A., Lerner J. et Scharfstein D. (2005), « Venture capital investment cycles : the impact of public markets », NBER Working Papers, 11385, May.

Gompers P. (2002), « Corporations and the Financing of Innovation : The Corporate Venturing Experience », Federal Reserve Bank of Atlanta Economic Review Fourth Quarter, pp. 1-19.

Gompers P. et Lerner J. (2001), « The Venture Capital Revolution », Journal of Economic Perspectives, vol. 15-2, Spring, pp. 145-168.

Gompers P. et Lerner J. (1998), « What drives venture capital fundraising ? » Brookings Proceedings on Microeconomic Activity, pp. 149-204.

Heiner R. (1983), « The origin of predictable behavior », American Economic Review, 73, 560-95.

Hodgson G., (1988), « Economics and Institutions », Polity Press, Cambridge.

Jeng L. et Wells P., (2000), « The determinants of venture capital funding : evidence across countries » Journal of Corporate Finance, vol. 6 pp. 241-289.

Kahneman D. et Tversky A., (1982), « Availability : a heuristic for judging frequency and probability », dans D. Kahneman, P. Slovic, et A. Tversky (eds), « Judgment Under Uncertainty : Heuristics and Biases », Cambridge, Cambridge University Press, 163-78.

Keynes J.-M., (1937) « The general theory of employment », Quarterly Journal of Economics, février, dans « Collected Writings XIV », Mac Millan, Londres, 1973, pp. 109-123.

Kester W.-C. (1984), « Today's options for tomorrow's growth », Harvard Business Review, 62(2), pp. 153-160.

Keynes J.-M. (1936), « Théorie générale de l'emploi, de l'intérêt et de la monnaie », Mac Millan, Londres, Traduction française : éd. Payot, Paris, 1979.

Knight F.H., (1921), « Risk, Uncertainty and Profit », Houghton Mifflin Company, Boston.

Lavoie M., (1992), « Foundations of Post-Keynesian Economic Analysis », Edward Elgar, Aldershot.

Laville F., (1998), « Modélisation de la rationalité limitée : de quels outils dispose-t-on? », Revue

Économique, vol. 49, n², pp. 335-365.

Lerner J. (2002), « Boom and Bust in the Venture Capital Industry and the Impact on Innovation », Federal Reserve Bank of Atlanta Economic Review Fourth Quarter, pp. 25-41.

Muth J.-F. (1961), « Rational expectations and the theory of price movements », Econometrica, July.

Myers S.-C., (1977), « Determinants of corporate borrowing », Journal of Financial Economics, vol. 5, pp. 147-175.

Neal P. (1996), « Keynesian uncertainty in credit markets », Journal of Post Keynesian Economics, printemps 2006, 18(3), pp. 397-418.

Portelli P. (1999), « Le développement du capital-risque en France », Les Notes Bleues de Bercy, 158, 1-15 mai.

Sahlman W.-A. (1990), « The structure and governance of venture capital organizations », Journal of Financial Economics, vol. 27, pp. 473-524.

Savage L.-J., (1954), « The Foundations of Statistics », John Wiley, New York.

Schertler A. (2003). « Driving Forces of Venture Capital Investments in Europe : A Dynamic Panel Data Analysis », Working Papers 1172, Kiel Institute for World Economics. 
Schwienbacher A. (2004), « An Empirical Analysis of Venture Capital Exits in Europe and in the United States, » Mimeo, University of Amsterdam.

Shackle G.-L.-S., (1955), « Uncertainty in Economics », Cambridge University Press, Cambridge.

Simon H. (1976), « From Substantive to Procedural Rationality », in Latsis S.-J. (ed.), « Method and Appraisal in Economics », Cambridge, Cambridge University Press, pp. 129-148.

Stigler G. (1961), « The Economics of Information », Journal of Political Economy, 69, pp. 213-225.

Trigeorgis L., (1996), « Real Options : Managerial Flexibility and Strategy in Resource Allocation », MIT Press, Cambridge.

Williamson O.-E. (1994), « Les institutions de l'Économie », Interéditions, Paris.

\section{NOTES}

1. Au cours de son développement, l'entreprise a besoin de capitaux propres pour financer sa croissance ou pour franchir une nouvelle étape (renforcement de la politique commerciale, réajustement au niveau de la production, lancement d'un nouveau produit, pénétration de marchés étrangers). Le capital-développement répond à ce type de besoins. Le capital-reprise/ transmission d'entreprise caractérise le financement d'opérations d'acquisitions d'entreprises. Les capital-investisseurs interviennent en effet fréquemment dans le cadre de financement de LBO (Leverage Buy Out). Ce type d'opération concerne quasi-exclusivement les entreprises «matures" car les LBO se caractérisent par des coefficients d'endettement importants et nécessitent donc, pour être réalisées, des flux de trésorerie les moins volatiles possibles.

2. Ces processus sont mis en place aussi bien avant (due diligence, syndication des investissements...) qu'après l'entrée dans le capital de l'entreprise. Les mécanismes de contrôle post-contractuels (attribution de bons de souscription d'actions au dirigeant, mise en place d'un financement par étapes...) visent à créer un cadre incitatif pour les dirigeants de manière à ce que leurs décisions de gestion soient conformes aux objectifs des actionnaires minoritaires que sont les capital-risqueurs.

3. Selon cette étude, l'investissement en capital-risque dans une firme ultérieurement introduite en bourse a un rendement moyen de $195 \%$ sur une période de 4,2 ans contre $40 \%$ pour une firme ultérieurement cédée.

4. Selon la terminologie employée dans l'approche du cycle de vie du produit. Les phases de conception et de lancement se caractérisent par des résultats durablement négatifs ainsi que par des taux d'échec des projets très élevés (de l'ordre de $70 \%$ pour la première, de 30 à $50 \%$ pour la seconde). Au total, seuls 15 à $20 \%$ des projets initiaux des start-up dépassent le stade de la phase de lancement (Adam et farber, 1994).

5. Ajoutons que, bien souvent, le seul élément « concret » qu'une start-up puisse présenter à un apporteur de capitaux externes est le plan de développement (business plan). Quelle que soit la qualité apportée à l'élaboration de ce document, il reste fondé quasi-exclusivement sur des projections incertaines et ne réunit donc pas les critères traditionnellement utilisés par les pourvoyeurs de fonds (solidité du bilan et rentabilité présente et passée, carnet de commandes, parts de marché...) pour filtrer les demandes de financement.

6. Pour reprendre la distinction utilisée par Lavoie (1992, p. 43), il y a risque lorsque chaque action mène à un ensemble de résultats possibles spécifiques dont la valeur est connue, chaque résultat étant associé à une probabilité spécifique ; il y a incertitude lorsque la probabilité d'un résultat est inconnue, ou lorsque la valeur d'un résultat est inconnue, ou lorsque certains des résultats d'une action sont inconnus, ou encore lorsque l'éventail des choix possibles est inconnu. 
7. Myers (1977), Kester (1984), Dixit et Pindyck (1994), Trigeorgis (1996), Amram et Kulatilaka (1999).

8. Les investissements en actifs réels et intangibles sont en partie irrécupérables (sunk costs) quand ils sont spécifiques à un type d'industrie ou à une firme (R\&D, marketing par exemple).

9. L'importance du financement par étapes en tant que mécanisme de contrôle de l'entreprise et de facteur de succès pour l'investissement en capital-risque a été démontrée empiriquement par différents auteurs, notamment par Sahlman (1990).

10. On utilise notamment le concept de VANA (valeur actuelle nette augmentée) qui est la somme de la valeur actuelle nette du projet et des options réelles qui lui sont attachées.

11. Par exemple, Laville (1998) distingue six familles de modèles de rationalité limitée : les modèles de satisfaction, de coûts, de fiabilité, d'automate, d'apprentissage et d'évolution.

12. Pour une application voisine, sur le marché bancaire, voir P. Neal (1996).

13. Cette idée était déjà présente chez Keynes pour qui : «la sagesse universelle enseigne qu'il vaut mieux pour sa réputation échouer avec les conventions que réussir contre elles » (1979, pp. 170).

14. Pour reprendre la position de Lucas lorsqu'il écrit : «En situation d'incertitude, le raisonnement économique ne sera d'aucune valeur » (Lucas, 1981, p. 224).

15. Pour l'année 2000, cette proportion est de l'ordre de $25 \%$ (AFIC 2005).

16. Ces dernières sont le plus souvent formulées en intégrant des asymétries d'information dans un environnement de simple risque probabilisable.

\section{RÉSUMÉS}

La littérature consacrée aux déterminants capital-risque insiste sur la relation positive existant entre le montant des fonds investis dans cette activité et la situation prévalant sur les marchés financiers. Cependant, au cours des dernières années, la relation entre marchés financiers et activité de capital-risque apparaît beaucoup moins claire: les comportements récents d'investissement des capitals-risqueurs semblent s'être sensiblement et durablement déconnectés de l'évolution des marchés financiers. Nous avons choisi d'expliquer ce phénomène en fondant notre analyse sur un élément essentiel mais généralement négligé dans la littérature : la forme spécifique de rationalité adoptée par les capital-risqueurs dans leurs choix d'investissement.

The literature dedicated to the determinants of venture capital insists on the positive relation existing between the amount of capital invested in this activity and the situation prevailing on financial markets. However, during last years, the relation between financial markets and venture capital investments appears much less clear: recent behaviors observed in venture capital markets seem to be strongly and durably disconnected from the evolution of financial markets. We chose to explain this phenomenon by basing our analysis on an essential - but generally neglected in the literature - element: the specific form of rationality adopted by venture capitalists in their investment decisions. 
INDEX

Mots-clés : Capital-risque, confiance, heuristique de disponibilité, rationalité limitée

Keywords : Availability Heuristics, Bounded Rationality, Confidence, Venture Capital

\section{AUTEURS}

\section{ERIC NASICA}

Université de Nice-Sophia Antipolis, DEMOS/GREDEG (UMR 6227 CNRS)

DOMINIQUE DUFOUR

Université de Nice-Sophia Antipolis, CRIFP 